\title{
Apoyo lingüístico inclusivo a alumnado no hispanohablante $y$ aprendizaje de competencias curriculares en Educación Primaria
}

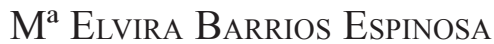

Dpto. de Didáctica de la Lengua y la Literatura, Universidad de Málaga Luis Morales Orozco

Modern Languages Department, United Nations International School (Nueva York)

Recibido: 27 de Marzo de 2011 / Aceptado 15 de Mayo de 2011

ISSN: 1697-7467

RESUMEN: El presente artículo expone un estudio orientado a investigar los efectos de un enfoque inclusivo al apoyo lingüístico a alumnado no hispanohablante dentro del programa de Aulas Temporales de Adaptación Lingüística (ATAL) en el rendimiento académico de alumando de Educación Primaria, en comparación con el efecto de una modalidad segregacionista del mismo. A tal fin, se esbozan los ejes teóricos que sustentaron la intervención dentro del aula y se muestran el diseño de la investigación, los resultados y su discusión, y las conclusiones que se derivan para orientar actuaciones en materia de aprovechamiento de recursos destinados al apoyo lingǘstico a alumnado no hispanohablante.

Palabras clave: Aulas Temporales de Adaptación Lingüística, aprendizaje de español como lengua vehicular, rendimiento académico, competencias curriculares, escuela inclusiva.

Inclusive Linguistic Support to Non-Spanish Speaking Pupils and the Learning of Curricular Competences in Primary Education

\begin{abstract}
This article presents a study aimed at investigating the effects of an inclusive modality of support to non-Spanish speakers within the programme of Linguistic Adaptation Temporary Classrooms on the academic performance of primary school learners, versus a 'pull-out' approach to such support. To this end, the theoretical tenets of the inclusive intervention programme are explained, together with the research design, the results and the discussion. In the last section, the main findings in favour of the inclusive approach are reviewed and some conclusions are derived for the allocation of resources to the linguistic support to non-Spanish speaking learners at schools.
\end{abstract}

Keywords: Linguistic Adaptation Temporary Classrooms, learning of Spanish as language of instruction, academic performance, curricular competences, inclusive school. 


\section{InTRODUCCIÓN}

La incorporación al sistema educativo de alumnado extranjero que carece de suficiente competencia lingüística en español ha generado en las administraciones educativas una serie de iniciativas y actuaciones específicas destinadas a la enseñanza de español como lengua vehicular. En la Comunidad Autónoma Andaluza, la medida con mayor impacto a nivel escolar son las Aulas Temporales de Adaptación Lingüística, puestas en marcha en el curso escolar 1997/1998 en dos centros de la provincia de Almería a modo de experiencia piloto que se extendió más tarde al resto de las provincias andaluzas (Ortiz, 2007).

La Orden de la Consejería de Educación de la Junta de Andalucía de 15 de enero de 2007 «por la que se regulan las medidas y actuaciones a desarrollar para la atención del alumnado inmigrante y, especialmente, las Aulas Temporales de Adaptación Lingüística» (en adelante, ATAL) define éstas como:

[...] programas de enseñanza y aprendizaje del español como lengua vehicular, vinculados a profesorado específico, que permiten la integración del alumnado inmigrante en el centro y su incorporación a los ritmos y actividades de aprendizaje propios del nivel en el que se encuentren escolarizados atendiendo a su edad y a su competencia curricular. (Artículo 5, punto 1)

Y asimismo establece que:

[...] estos programas deberán realizarse en el aula ordinaria. Se podrán organizar grupos de apoyo atendidos por profesorado específico para llevar a cabo la atención fuera del aula ordinaria cuando circunstancias especiales en la comprensión-expresión de la lengua española así lo aconsejen. (Artículo 5, punto 2)

Sin embargo, a tenor de la organización de estos programas en los centros escolares andaluces, estas «circunstancias especiales» parecen darse en la inmensa mayoría de los casos, puesto que la forma más frecuente de atención al alumnado extranjero en su proceso de aprendizaje del español es atenderlo fuera de su aula de referencia.

Este modelo de atención externa es llevado a cabo de forma institucional en otras Comunidades Autónomas como Madrid, por ejemplo, a través de sus Aulas de Enlace, aunque ya hay algunos trabajos de investigación (p. ej., García Fernández y Moreno Herrero, 2003; García Fernández, Moreno Herrero y Sánchez Delgado, 2008; Ortiz, 2006) que han concluido que este tipo de agrupamientos promueve un modelo escolar homogeneizador y competitivo, con resistencia a aceptar la diversidad y que, además, favorece que el resto del profesorado, ajeno a estas aulas específicas, no asuma plenamente su responsabilidad en la adquisición de competencia lingüística en español del alumnado que se incorpora al Centro sin poseerla, lo cual, en nuestro contexto, contradice el espíritu de la Orden antes mencionada que especifica que «cada una de las actuaciones a desarrollar con el alumnado inmigrante corresponde a la totalidad del profesorado del centro» ${ }^{1}$.

\footnotetext{
1 Artíulo 2, punto 3 .
} 
Ma Elvira Barrios Espinosa y Luis Morales Orozco Apoyo lingüístico inclusivo a alumnado...

Frente a esta modalidad imperante ${ }^{2}$, que, conforme a la normativa aludida, permitiría -aunque, insistimos, únicamente en casos excepcionales- la asistencia de alumnado extranjero con dificultades en español a grupos de apoyo organizados fuera de su aula ordinaria un máximo de 10 horas $^{3}$ en Educación Primaria y de 15 horas en Educación Secundaria y durante un periodo máximo de dos cursos escolares, se propone un enfoque alternativo de atención en su propia aula basado en principios de la educación inclusiva e intercultural y del aprendizaje cooperativo, en la atención a los factores afectivos y en una concepción de la enseñanza de la lengua del país de acogida compatible con una defensa del bilingüismo de ganancia (Morales Orozco, 2006, 2007, 2009).

Esta modalidad inclusiva supone que, el alumnado con dificultades en español para seguir el currículo ordinario, en lugar de salir de su aula de referencia durante unas horas a la semana (hasta un $40 \%$ del horario lectivo mínimo en Educación Primaria) para recibir apoyo lingüístico, permanece en ella la totalidad del tiempo y es el docente de ATAL quien acude al aula de referencia a prestar ese apoyo; esto se traducía, en el centro en el que se desarrolló la investigación, en que, en una misma aula coincidían dos docentes simultáneamente, el docente ordinario y el docente de ATAL, durante una media de 3 horas semanales, durante 4 cursos escolares, al irse incorporando en dichos grupos, cada nuevo curso y durante el curso, alumnado necesitado de apoyo.

Varios autores han abordado los fundamentos educativos conceptuales e ideológicos diferenciados -e incluso, antagónicos- que sustentan una y otra visión de este tipo de apoyo lingüístico en el entorno escolar (p. ej., Bonal, 2004; Jiménez Gámez, 2004; Ortiz, 2006; Quintana, 2003); ahora bien, no obstante el interés y relevancia de discusiones que contraponen ambas alternativas en competencia a la enseñanza del español como lengua vehicular, las cuales evidencian las concepciones educativas conceptuales e ideológicas subyacentes para concluir subrayando, generalmente, la superioridad ética del enfoque inclusivo frente al que segrega en aulas específicas, cabe preguntarse, además, por la eficiencia de ambas modalidades para garantizar su cometido de posibilitar al alumnado la adquisición de competencias establecidas en el currículo escolar.

Entendemos que esta dimensión de la investigación sobre formatos de suministro de apoyo lingüístico centrada en el rendimiento académico, no abordada hasta la fecha, es clave para orientar políticas e iniciativas en el ámbito de la atención al alumnado con problemas de acceso al currículo ordinario a causa de su insuficiente competencia en español.

En este sentido, el presente artículo se propone presentar una investigación destinada a contrastar la eficiencia de un enfoque inclusivo al apoyo lingüístico -en contraposición a la eficiencia de la modalidad segregacionista extendida de forma generalizadapara el aprendizaje de competencias curriculares de alumnado extranjero con dificultades

2 En el estudio sobre el programa de ATAL en la provincia de Cádiz realizado durante el curso 2007-2008, únicamente el 6,2\% de los profesores de ATAL encuestados manifestaron que desarrollaban apoyos en el aula de referencia en alguna ocasión.

3 Esto representa un $40 \%$ del horario lectivo mínimo obligatorio en esta etapa escolar. 
en español en un mismo centro escolar. Más concretamente, el artículo expone un estudio $^{4}$ encaminado a investigar el efecto de un enfoque inclusivo al apoyo lingüístico en el rendimiento académico de grupos de clase que lo han experimentado durante un periodo de 4 cursos de Educación Primaria, a razón de una media de 3 horas semanales, en comparación con el que produce una modalidad segregacionista en el mismo centro escolar. A tal fin, se esbozan los ejes teóricos que sustentaron la intervención de apoyo lingüístico dentro del aula de referencia del alumnado beneficiario del apoyo ATAL y se exponen el diseño de la investigación, los resultados y su discusión, y las conclusiones que se derivan para orientar políticas, actuaciones y directrices en materia de aprovechamiento de recursos destinados al apoyo lingüístico de alumnado no hispanohablante en centros con financiación pública.

\section{FUNDAMENTACIÓN TEÓRICA DE LA INTERVENCIÓN INCLUSIVA DE APOYO ATAL}

Como se señaló en el apartado anterior, la modalidad inclusiva de apoyo lingüístico que se sometía a investigación, desarrollada en el Colegio de Infantil y Primaria «El Chaparral» durante los cuatro cursos académicos previos al estudio e impulsada por el profesor de ATAL, se sustentaba en cinco pilares teóricos, a saber, el modelo intercultural como ideal para seguir en contextos educativos multiculturales, una concepción del bilingüismo promovido en el alumnado como bilingüismo de ganancia, la importancia de la motivación en todo proceso de aprendizaje, la inclusión frente a la segregación en educación, y el aprendizaje cooperativo como herramienta indispensable de trabajo en el aula.

\subsection{Modelo intercultural}

La intervención ATAL asumía el modelo intercultural como un enfoque educativo basado en el respeto y la apreciación cultural, que se orienta al conjunto de los miembros de la comunidad educativa y que afecta a todas las dimensiones del proceso educativo. Ello implica:

- eludir el etnocentrismo, entendido este como una forma simplista y tendenciosa de contemplar las relaciones entre las comunidades basada en la mitificación o universalización de los modelos culturales propios (Trujillo, 2006);

- evitar la consideración del alumnado con lengua materna diferente al de acogida como alumnado con dificultades intelectuales, valorando además su propia len-

${ }^{4}$ El estudio que aquí se presenta forma parte de una investigación más amplia en la que, junto a datos sobre rendimiento académico, se utilizaron datos observacionales de clases, entrevistas y encuestas para explorar estrategias docentes y dinámicas de aula, así como perspectivas de profesorado y alumnado en torno a la modalidad inclusiva del apoyo ATAL; esta investigación, titulada «Integración lingüística y bilingüismo de ganancia en contextos escolares inclusivos», que recibió una ayuda de la Consejería de Educación de la Junta de Andalucía para proyectos de investigación educativa para el año 2008 (referencia: PIV-057/08), fue desarrollada a lo largo del curso escolar 2008-2009 y coordinada por Luis Morales Orozco. 
Ma Elvira Barrios Espinosa y Luis Morales Orozco Apoyo lingüístico inclusivo a alumnado...

gua y su bagaje cultural como potencialmente enriquecedores para el resto de la comunidad educativa; y,

- valorar el contexto multicultural como espacio para promover la enseñanza de habilidades sociales que favorecen la superación de la xenofobia, el racismo, el sexismo, etc.

\subsection{Bilingüismo de ganancia o bilingüismo aditivo}

Una de las ideas inspiradoras del enfoque llevado a cabo en el centro escolar en el que se sitúa la investigación es la relativa al bilingüismo de ganancia o bilingüismo aditivo.

Según Lambert (1975), el bilingüismo aditivo es el que resulta de un programa en el que el alumnado mantiene su primera lengua a la vez que adquiere su segunda lengua. Este tipo de programas se caracteriza por procurar el desarrollo de esa primera lengua y valorar la cultura de origen del estudiante, a la vez que este va incoporando una segunda lengua a su repertorio lingüístico. En una situación de bilingüismo de ganancia, es improbable que esta incorporación comporte el que la lengua y la cultura de origen sean reemplazadas o desplazadas (Lambert, 1980, en Baker, 2001: 58).

Baker (2001: 114) alude a la potencial influencia ambiental en la generación de modalidades de bilingüismo diferenciadas cuando afirma:

Cuando una segunda lengua y cultura han sido adquiridas con poca o ninguna presión para sustituir o reducir la primera lengua, puede darse una forma aditiva de bilingüismo. Cuando una segunda lengua y cultura han sido adquiridas con presión para reemplazar o denigrar a la primera lengua, puede producirse una forma substractiva de bilingüismo. ${ }^{5}$

Cummins (2000), para quien el término bilingüismo de ganacia «se refiere a la forma de bilingüismo que resulta cuando los estudiantes añaden una segunda lengua a su equipo de herramientas, mientras que continúan desarrollándose conceptual y académicamente en su primera lengua» (p. 37), afirma que, en los últimos 30 años del siglo anterior, existía ya un número cercano a los 150 estudios que establecían la «asociación positiva entre bilingüismo de ganancia y desarrollo lingüístico, cognitivo o académico de los estudiantes» (p. 37), los cuales sugieren la importante influencia que puede ejercer en su desarrollo académico y conceptual la competencia alcanzada por el estudiante en sus dos lenguas.

En el caso de alumnado no hispanohablante que se incorpora al sistema educativo en pleno proceso de adquisición de su primera lengua, la falta de oportunidades para avanzar su competencia en la primera lengua puede afectar muy negativamente a su progreso intelectual y a su propia imagen. Como afirma Martín Rojo (1995: 313):

5 Traducción de los autores. 
El bilingüismo de pérdida o de sustitución se desarrolla cuando las lenguas no son complementarias, sino que concurren, de manera que esta forma se produce cuando una comunidad rechaza sus propios valores socioculturales en beneficio de una lengua que desde los valores dominantes cuenta con prestigio cultural y económico. El resultado puede ser que la lengua materna se deteriore y sea sustituida por la segunda lengua, o bien, como ocurre frecuentemente, que ninguna de ellas se adquiera plenamente. Ambas situaciones se producen con frecuencia entre los inmigrantes que son escolarizados en la lengua del país de acogida. Lo que conlleva el que se produzca una etapa intermedia de privación de la competencia en las dos lenguas (Lambert 1977, p. 19), que puede prolongarse. Esta privación se manifiesta a todos los niveles e influye en el desarrollo cognitivo y de la personalidad.

En consecuencia, los beneficios que parecen derivarse de un bilingüismo de ganancia apoyan la postura de favorecer el matenimiento de la primera lengua de niños no hispanohablantes en nuestro sistema educativo, no únicamente en su ámbito familiar, sino también en los centros escolares, como instrumento con el que procurarles recursos intelectuales y académicos.

\subsection{Motivación}

La motivación del alumnado hacia la escuela y las tareas de aprendizaje representaba en esta intervención didáctica otro de los pilares en los que se sustentaba; dicha motivación pretendía favorecerse a través de tres vías:

- la actitud del enseñante, quien conscientemente procuraba esforzarse en trabajar la relación afectiva con el alumnado ya que, según demuestran algunos estudios, la incidencia del comportamiento disruptivo o desinteresado es menor en las aulas cuyos profesores suelen mostrar una serie de características afectivas positivas (Barrios y García Mata, 2006);

- las formas de enseñar, que procuraban presentar el trabajo por realizar de manera tal que despertara la curiosidad y el interés, fomentara la participación del alumnado y valorara el esfuerzo realizado y no solo los resultados obtenidos; y,

- la introducción del componente lúdico en el proceso de aprendizaje como forma de crear un espacio de confianza, libertad y creatividad donde el alumnado juega sin percatarse de que también aprende simultáneamente; además, se partía de la convicción de que el juego «tiene un enorme potencial como herramienta que facilita el acercamiento, conocimiento y enriquecimiento entre personas de diferentes culturas» (García Marín y Martínez Ten, 2004: 14).

\subsection{Inclusión frente a segregación}

En un contexto inclusivo las diferencias se perciben como tales y no como déficits y carencias (Morales Orozco, 2006) y se entiende que todos los niños y niñas pertenecen al grupo y pueden aprender de una escuela en la que la diversidad se conciba como un valor añadido (Stainback y Stainback, 1999). Se parte de la convicción de que la educación inclusiva, aplicada a la educación en general y no solo al alumnado con necesidades educativas, tiene ventajas evidentes: 
Ma Elvira Barrios Espinosa y Luis Morales Orozco Apoyo lingüístico inclusivo a alumnado...

- se elimina la estigmatización del alumnado por motivos cualesquiera;

- se elevan las expectativas del alumnado de forma global, ya que no hay nadie específico señalado como necesitado de apoyo: es el grupo el que recibe el apoyo; desde esta perspectiva, se entiende que las necesidades de unos se equilibran con las necesidades de los otros;

- mejora el aprendizaje al reforzarse la autoestima del alumnado;

- favorece el clima escolar positivo;

- hace replantearse la eficacia de modelos educativos hechos a la medida del alumno 'medio', figura inexistente más allá de en los libros de texto; y,

- el contacto continuo con iguales que hablan una lengua diferente favorece el bilingüismo de ganancia (Martín Rojo, 2004).

\subsection{Aprendizaje cooperativo}

El aprendizaje cooperativo es un modelo de aprendizaje tangencialmente opuesto al que impera en la mayoría de los espacios educativos en los que la responsabilidad del proceso de enseñanza y aprendizaje recae casi exclusivamente en el profesorado y en los que predomina un modelo pedagógico basado en la competitividad y en el individualismo. La filosofía cooperativa nos permite aprovechar plenamente la interacción entre los alumnos para que todos alcancen los objetivos curriculares al máximo de sus posibilidades y descubran que trabajando juntos se consigue más que trabajando de manera aislada (Díaz-Aguado, 2006). Y, además:

- el alumnado aprende que todos los miembros del grupo pueden aportar algo al esfuerzo común, con lo que aumentará su autoestima al sentirse apoyados unos por otros;

- un alumno «docente» puede ser más eficaz a la hora de explicar determinados conceptos a sus iguales; y,

- el uso de diferentes lenguas en el aula se verá potenciado (Díaz-Aguado, 2006).

\section{Diseño de la inVestigación}

\subsection{Objetivos e interrogantes}

Los objetivos que se planteó el presente estudio fueron los siguientes:

a. Comprobar la eficiencia para el aprendizaje de competencias curriculares de un enfoque inclusivo al apoyo a alumnado extranjero con dificultades lingüísticas en español dentro del aula de referencia de este alumnado.

b. Contrastar el rendimiento de alumnado extranjero con dificultades lingüísticas que ha recibido apoyo dentro del aula de referencia con el rendimiento de alumnado con dificultades lingüísticas que ha recibido apoyo fuera del aula de referencia, que es el enfoque que prevalece en los centros escolares andaluces.

c. Conocer el efecto de ambos enfoques al apoyo lingüístico en el rendimiento académico del alumnado hispanohablante. 
d. Ofrecer algunas consideraciones de interés organizativo relativas a políticas y actuaciones en torno al apoyo lingüístico a alumnado extranjero en centros educativos sostenidos con fondos públicos.

Más concretamente, la investigación pretendía dar respuesta a los siguientes interrogantes:

1. En la Prueba de la Evaluación de Diagnóstico que realiza la Consejería de Educación de la Junta de Andalucía, ¿obtiene el alumnado de grupos de clase en los que se ha aplicado un enfoque inclusivo al apoyo ATAL un rendimiento académico significativamente superior que el alumnado de grupos en los que no se ha aplicado esta modalidad de apoyo lingüístico?

2. ¿Qué conjunto de alumnado se beneficia de una modalidad inclusiva de apoyo ATAL en términos de aprendizaje de competencias curriculares, aquel cuya lengua materna es el español y/o aquel cuya lengua materna no es el español?

3. ¿De cuál de las dos modalidades de apoyo lingüístico se beneficia más el alumnado cuya lengua materna es el español en términos de aprendizaje de competencias curriculares?

4. A la luz de los resultados, ¿cuál de las dos modalidades resulta más eficiente para favorecer el aprendizaje de competencias curriculares?

\subsection{Contexto}

La investigación se llevó a cabo en el CEIP El Chaparral (Mijas, Málaga), en el que la mayoría del alumnado escolarizado, un $60,5 \%$, es de nacionalidad diferente a la española y, de este porcentaje, un $82 \%$ tiene una lengua materna diferente al español. Un total de 16 lenguas maternas diferentes coexisten en el colegio, aunque el inglés es la predominante, bien como lengua materna (de un $48 \%$ del alumnado aproximadamente), bien como L2 en el caso de alumnado procedente de países del Norte de Europa ${ }^{6}$.

Desde el curso 2004-2005, la mayoría de los tutores, en colaboración con el profesor de ATAL, decidieron intentar llevar a cabo el apoyo lingüístico dentro del aula de referencia y ya desde el primer año los resultados fueron prometedores, como lo demuestran los datos obtenidos en la investigación-acción realizada en el centro sobre este tipo de apoyo (Morales Orozco, 2006).

\subsection{Participantes}

Tomaron parte en la investigación un total de 67 alumnos y alumnas escolarizados en los cuatro grupos de $5^{\circ}$ curso del centro. En el curso en el que se realizó el estudio, en dos de ellos se llevaba realizando el apoyo al alumnado con dificultades lingüísticas dentro del aula desde el curso 2004-2005, mientras que en los dos restantes dicho apoyo se realizaba en el aula específica de ATAL (Tabla 1).

\footnotetext{
${ }^{6}$ Los datos aportados se refieren a la situación del centro en el mes octubre de 2009.
} 
Ma Elvira Barrios Espinosa y Luis Morales Orozco Apoyo lingüístico inclusivo a alumnado...

Tabla 1. Distribución del alumnado participante según su lengua materna, curso y tipo de apoyo ATAL que se practicaba en el mismo.

\begin{tabular}{|c|c|c|c|c|c|c|}
\hline & \multicolumn{2}{|c|}{$\begin{array}{l}\text { Apoyo ATAL } \\
\text { fuera del aula }\end{array}$} & \multirow[t]{2}{*}{ Total } & \multicolumn{2}{|c|}{$\begin{array}{l}\text { Apoyo ATAL } \\
\text { dentro del aula }\end{array}$} & \multirow[t]{2}{*}{ Tota } \\
\hline & $5^{\circ} \mathrm{A}$ & $5^{\circ} \mathrm{D}$ & & $5^{\circ} \mathrm{B}$ & $5^{\circ} \mathrm{C}$ & \\
\hline $\begin{array}{l}\text { Español como lengua } \\
\text { materna }\end{array}$ & 3 & 7 & 10 & 3 & 7 & 10 \\
\hline $\begin{array}{l}\text { Inglés como lengua } \\
\text { materna }\end{array}$ & 7 & 6 & 13 & 6 & 6 & 12 \\
\hline Otras lenguas maternas & 7 & 5 & 12 & 6 & 4 & 10 \\
\hline Total & 17 & 18 & 35 & 15 & 17 & 32 \\
\hline
\end{tabular}

\subsection{Herramientas de obtención de datos}

Para los fines del estudio se contó con los resultados obtenidos por los participantes en la Prueba de la Evaluación de Diagnóstico que, desde el curso 2006-2007, la Consejería de Educación obliga a administrar cada año a los centros escolares andaluces financiados con fondos públicos; en el curso académico 2008-2009, al que pertenecen los datos, las pruebas se administraron a principios de octubre al alumnado de $5^{\circ}$ curso.

La Prueba de la Evaluación de Diagnóstico del curso académico referido comprendía tres pruebas diferenciadas, destinadas a evaluar la competencia del alumnado en Comunicación lingüística (Lengua Española), en Matemáticas y en Conocimiento e interacción con el medio físico y natural. La prueba que evaluaba la competencia en Comunicación lingüística constaba de 27 ítems, la de Matemáticas, de 18, y la de Conocimiento e interacción con el medio físico y natural, de $24^{7}$.

Los datos empleados, los cuales figuraban en el informe de competencias individualizado y de grupo que remitió al Centro la Dirección General de Ordenación y Evaluación Educativa, responsable de la regulación de dichas Pruebas de Evaluación de Diagnóstico y del establecimiento del procedimiento de aplicación de las mismas, fueron los que a continuación se detallan:

a) las puntuaciones directas individuales ${ }^{8}$ que obtuvo cada alumno en las pruebas de Comunicación lingüística (Lengua Española), Matemáticas y Conocimiento e interacción con el medio físico y natural; y,

b) los resultados obtenidos por cada alumno en las dimensiones de 'Comprensión oral', 'Comprensión lectora' y 'Comprensión escrita' que la prueba de competencia en Comunicación lingüística comprendía.

${ }^{7}$ La Resolución de 30 de julio de 2008, de la Dirección General de Ordenación y Evaluación Educativa, por la que se desarrollan determinados aspectos de la Orden anterior, regula las Pruebas de la Evaluación de Diagnóstico y establece el procedimiento de aplicación en los centros docentes de Andalucía sostenidos con fondos públicos (BOJA núm. 161, de 13 de agosto de 2008).

${ }^{8}$ La puntuación directa individual se obtiene sumando las puntuaciones registradas en cada ítem de la prueba como resultado de aplicar a la respuesta dada por el alumno o alumna los criterios para la corrección establecidos. 
Un total de 67 alumnos realizaron la prueba básica en Comunicación lingüística, de 61, la prueba de competencia en Matemáticas y de 65, la que valoraba la competencia en el Conocimiento e interacción con el medio físico y natural.

Las Pruebas de Evaluación de Diagnóstico tuvieron lugar los días 6 (Prueba de competencia básica en Matemáticas), 7 (Prueba de competencia básica en Comunicación lingüística (Lengua Española) y 8 (Prueba de competencia básica en el Conocimiento y la interacción con el mundo físico y natural) de octubre de 2008.

Además, se obtuvieron datos biográficos de los participantes relativos a su lengua materna.

\subsection{Procedimientos de análisis de los datos}

Para el análisis de los datos se calcularon los estadísticos descriptivos de tendencia central (media, mediana, moda) y de dispersión (desviación típica, mínimo y máximo) del rendimiento académico del alumnado, entendido este en término de los resultados obtenidos por los participantes en las pruebas de las tres competencias básicas evaluadas por la Prueba de Diagnóstico (Comunicación lingüística, Matemáticas y Conocimiento e interacción con el mundo físico y natural) y en las dimensiones de Comprensión oral, Comprensión lectora y Expresión escrita de la prueba de Comunicación Lingüística.

Para efectuar comparaciones entre grupos (alumnado en cuya tutoría se desarrollaba un enfoque inclusivo al apoyo ATAL frente a alumnado en cuya tutoría se desarrollaba un enfoque segregacionista al apoyo ATAL, y alumnado hispanohablante frente alumnado de lengua materna distinta al español) en relación al rendimiento en dichas pruebas, se aplicó la prueba U de Mann-Whitney. En todos los casos se ha trabajado con un nivel de confianza del $95 \%$.

\section{Resultados y discusión}

\subsection{Rendimiento en la Prueba de Diagnóstico: Grupo en tutoría con apoyo ATAL dentro del aula frente a grupo en tutoría con apoyo ATAL fuera del aula de referencia}

\subsubsection{Rendimiento en la 'Prueba de competencias básicas en Comunicación lingüistica (Lengua Española)' y en dimensiones de la misma}

La 'Prueba de competencia básica en Comunicación lingüística (Lengua Española)' administrada en el curso académico 2008-2009 en los centros públicos y concertados de Educación Primaria de Andalucía comprendía un total de 27 ítems, de los cuales, 7 medían capacidades de comprensión oral, otros 13, capacidades de comprensión lectora y, los 7 restantes, capacidades de expresión escrita.

En relación al rendimiento en la Prueba de competencias básicas en comunicación lingüística (rango posible: de 27 a 108 puntos) y en las tres dimensiones que la integran ('Comprensión oral', 'Comprensión lectora' y 'Expresión escrita') (rango posible: de nivel 1 a nivel 4), el grupo en el que se recibe el apoyo ATAL dentro del aula consigue un 
Ma Elvira Barrios Espinosa y Luis Morales Orozco Apoyo lingüístico inclusivo a alumnado...

rendimiento medio superior, como puede constatarse en los datos que figuran en la columna de 'Media' de las Tablas 2 y 3, que, en la prueba general, representa una diferencia de algo más de 6 puntos. Asimismo, los datos de la mediana constatan que el $50 \%$ del alumnado que pertenece al grupo en el que se suministra el apoyo ATAL dentro del aula rinde en una medida sensiblemente superior al mismo porcentaje en el grupo en el que dicho apoyo se produce fuera del aula de referencia.

Tabla 2. Resultados del análisis estadístico descriptivo de la variable: Rendimiento en la 'Prueba de competencias básicas en Comunicación lingüistica'.

\begin{tabular}{|l|l|l|l|l|l|l|l|}
\hline \multicolumn{1}{|c|}{ Grupo } & $\mathrm{N}$ & Media & Mediana & Moda & $\begin{array}{l}\text { Desv. } \\
\text { típica }\end{array}$ & Mín. & Máx. \\
\hline $\begin{array}{l}\text { Tutoría con apoyo } \\
\text { ATAL dentro del } \\
\text { aula }\end{array}$ & 33 & 55,24 & 57,00 & $59^{\mathrm{a}}$ & 11,068 & 29 & 77 \\
\hline $\begin{array}{l}\text { Tutoría con apoyo } \\
\text { ATAL fuera del } \\
\text { aula }\end{array}$ & 34 & 48,91 & 45,50 & 49 & 13,697 & 30 & 77 \\
\hline
\end{tabular}

a. Existen varias modas. Se muestra el menor de los valores

Tabla 3. Resultados del análisis estadístico descriptivo de las variables: Rendimiento en 'Comprensión oral', 'Comprensión lectora' y 'Expresión escrita' en la 'Prueba de competencias básicas en Comunicación lingüística'.

\begin{tabular}{|c|c|c|c|c|c|c|}
\hline & Grupo & $\mathrm{N}$ & Media & Mediana & Moda & $\begin{array}{l}\text { Desv. } \\
\text { típica }\end{array}$ \\
\hline \multirow{2}{*}{ 言 정 } & $\begin{array}{l}\text { Apoyo ATAL } \\
\text { dentro del aula }\end{array}$ & 33 & 2,82 & 3,00 & 3 & 1,185 \\
\hline & $\begin{array}{l}\text { Apoyo ATAL } \\
\text { fuera del aula }\end{array}$ & 34 & 2,24 & 2,00 & 1 & 1,208 \\
\hline \multirow{2}{*}{ 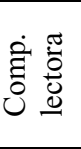 } & $\begin{array}{l}\text { Apoyo ATAL } \\
\text { dentro del aula }\end{array}$ & 33 & 2,88 & 3,00 & 3 & 893 \\
\hline & $\begin{array}{l}\text { Apoyo ATAL } \\
\text { fuera del aula }\end{array}$ & 34 & 2,41 & 2,00 & 2 & ,925 \\
\hline \multirow{2}{*}{ 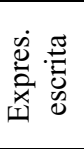 } & $\begin{array}{l}\text { Apoyo ATAL } \\
\text { dentro del aula }\end{array}$ & 33 & 2,03 & 2,00 & 1 & 1,045 \\
\hline & $\begin{array}{l}\text { Apoyo ATAL } \\
\text { fuera del aula }\end{array}$ & 34 & 1,85 & 1,00 & 1 & 1,209 \\
\hline
\end{tabular}

La comparación de los dos grupos con respecto a las anteriores cuatro variables de rendimiento mediante la prueba $U$ de Mann-Whitney revela que existen diferencias significativas entre ellos en relación a tres de estas variables ('Prueba de competencia básica en Comunicación lingüística $(\mathrm{p}=0,021)$, dimensión de 'Comprensión oral' $(\mathrm{p}=0,036)$ y dimensión de 'Comprensión lectora' $(\mathrm{p}=0,015)$. 
En relación a la cuarta variable de rendimiento relativa a la competencia lingüística, la dimensión de 'Expresión escrita', no se encontraron diferencias estadísticamente significativas entre ambos grupos; se daba la circusntancia de que de los 7 ítems que medían capacidades de expresión escrita, 4 de ellos figuraban en la parte final de la prueba, lo que puede explicar que un elevado número de alumnos, por falta de tiempo, dejara sin responder esos últimos ítems de la prueba (el valor de la moda, en ambos grupos, como se indica en la Tabla 3, es 1, nivel que, en las pautas de corrección oficiales de la prueba, incluye la ausencia de respuesta). Entendemos, por consiguiente, que esta circunstancia invalida en gran medida los datos empleados en el estudio en materia de esta dimensión y puede ser la causa, tanto de que no se encuentren diferencias significativas en relación a la 'Expresión escrita' entre ambos grupos como del resto de los resultados que obtiene el estudio referidos a esta dimensión, los cuales, como se explica más adelante, difieren generalmente de la tendencia seguida por los resultados referidos a las otras competencias y dimensiones evaluadas.

Se comprueba, por consiguiente, que, en la 'Prueba de competencias básicas en Comunicación lingüística (Lengua Española)' y en tres de las cuatro dimensiones que dicha prueba comprendía, el grupo de alumnos que pertenece a aulas con una modalidad inclusiva de apoyo lingüístico obtiene unos resultados superiores a los obtenidos por el grupo en el que dicho apoyo se produce fuera del aula, y que las diferencias son estadísticamente significativas.

\subsubsection{Rendimiento en la 'Prueba de competencia básica en Matemáticas'}

Con respecto a la prueba de competencia en matemáticas (rango posible: de 18 a 72 puntos), se procedió, al igual que en el caso anterior, a calcular los estadísticos descriptivos de grupo (Tabla 4), en los que se aprecia un rendimiento medio superior en esta materia en el grupo que participa en el proyecto inclusivo, que supera los 10 puntos de diferencia en el valor del promedio y 17,70 en relación al valor de la mediana.

Tabla 4. Resultados del análisis estadístico descriptivo de la variable: Rendimiento en la 'Prueba de competencia básica en Matemáticas'.

\begin{tabular}{|l|c|c|c|c|c|c|c|}
\hline \multicolumn{1}{|c|}{ Grupo } & $\mathrm{N}$ & Media & Mediana & Moda & $\begin{array}{c}\text { Desv. } \\
\text { típica }\end{array}$ & Mín. & Máx. \\
\hline $\begin{array}{l}\text { Tutoría con apoyo } \\
\text { ATAL dentro del } \\
\text { aula }\end{array}$ & 28 & 46,50 & 49,50 & 36 & 12,468 & 18 & 63 \\
\hline $\begin{array}{l}\text { Tutoría con apoyo } \\
\text { ATAL fuera del aula }\end{array}$ & 33 & 36,06 & 32,00 & $30^{\mathrm{a}}$ & 11,776 & 18 & 64 \\
\hline
\end{tabular}

a. Existen varias modas. Se muestra el menor de los valores

La comparación de los dos grupos con respecto a esta medida de rendimiento mediante la prueba $U$ de Mann-Whitney revela que existen diferencias significativas entre ellos $(\mathrm{p}=0,001)$. 
Ma Elvira Barrios Espinosa y Luis Morales Orozco Apoyo lingüístico inclusivo a alumnado...

Se constata, por consiguiente, que, en la 'Prueba de competencia básica en Matemáticas', el grupo de alumnos que pertenece a aulas con una modalidad inclusiva de apoyo lingüístico obtiene unos resultados superiores a los obtenidos por el grupo en el que dicho apoyo se produce fuera del aula y que la diferencia es estadísticamente significativa.

\subsubsection{Rendimiento en la 'Prueba de competencia básica en el Conocimiento y la interacción con el mundo físico y natural'}

Los resultados obtenidos en la 'Prueba de competencia en conocimiento e interacción con el medio físico y natural (rango posible: de 24 a 96 puntos), confirman de nuevo el rendimiento superior del grupo formado por los cursos en los que se aplica un enfoque inclusivo al apoyo lingüístico (ver Tabla 5), el cual representa una diferencia de más de 8 puntos en el valor de la media y de 16 en relación al valor de la mediana.

Tabla 5. Resultados del análisis estadístico descriptivo de la variable: Rendimiento en la 'Prueba de competencia básica en el Conocimiento y la interacción con el mundo físico y natural'.

\begin{tabular}{|l|c|c|c|c|c|c|c|}
\hline \multicolumn{1}{|c|}{ Grupo } & $\mathrm{N}$ & Media & Mediana & Moda & $\begin{array}{c}\text { Desv. } \\
\text { típica }\end{array}$ & Mín. & Máx. \\
\hline $\begin{array}{l}\text { Tutoría con apoyo } \\
\text { ATAL dentro del } \\
\text { aula }\end{array}$ & 32 & 50,22 & 52,00 & $40^{\mathrm{a}}$ & 10,102 & 25 & 69 \\
\hline $\begin{array}{l}\text { Tutoría con apoyo } \\
\text { ATAL fuera del aula }\end{array}$ & 33 & 41,70 & 36,00 & 34 & 11,414 & 27 & 66 \\
\hline
\end{tabular}

a. Existen varias modas. Se muestra el menor de los valores.

Asimismo, la comparación de los dos grupos mediante la prueba U de MannWhitney revela que existen diferencias significativas entre ellos $(p=0,002)$.

Se constata, por consiguiente, que, en la 'Prueba de competencia básica en el Conocimiento y la interacción con el mundo físico y natural', el grupo de alumnos que pertenece a aulas con una modalidad inclusiva de apoyo lingüísticos obtiene unos resultados superiores, y que la diferencia es estadísticamente significativa.

En conclusión, según se desprende de los resultados en el conjunto de las competencias que valoran las pruebas de evaluación de diagnóstico elaboradas por la Dirección General de Ordenación y Evaluación Educativa de Consejería de Educación de la Junta de Andalucía, el grupo de alumnos en cuyas tutorías el apoyo ATAL se desarrolla dentro del aula de referencia consigue un rendimiento académico superior al que obtiene el grupo conformado por el alumnado en el que, en sus tutorías, dicho apoyo se produce fuera del aula de referencia.

En relación a los resultados expuestos hasta aquí, ha de tenerse en cuenta que este rendimiento superior en las tres competencias analizadas se refiere al grupo en su conjunto, no al subgrupo conformado por alumnado no hispanohablante, lo que parece sugerir que se trata de una modalidad de apoyo que beneficia globalmente al alumnado 
de las tutorías en las que el docente de ATAL proporciona dicho apoyo dentro del aula en la que existe alumnado con dificultades lingüísticas.

Es razonable conjeturar que la presencia dentro del aula del docente de ATAL, una media de 3 horas semanales durante 4 cursos escolares, puede haber contribuido a este rendimiento superior demostrado por el alumnado en grupos en el que el apoyo ATAL se realiza dentro del aula en relación a la totalidad de las competencias evaluadas; además, como se ha evidenciado, este rendimiento superior no lo es únicamente en competencia lingüística, sino, además, en Matemáticas y en el Conocimiento y la interacción con el mundo físico y natural. Se confirma, por consiguiente, que un apoyo ATAL inclusivo -frente a la modalidad segregacionista- favorece en mayor grado la adquisición de competencias curriculares del conjunto del alumnado.

\subsection{Rendimiento en la Prueba de Diagnóstico: Alumnado con español como LM frente a alumnado con una LM diferente al español}

Una vez establecido que el alumnado que pertenece a una tutoría en la que el apoyo ATAL se realiza de manera inclusiva obtiene un rendimiento superior en pruebas que evalúan sus competencias en Comunicación Lingüística, Matemáticas y Conocimiento e interacción con el mundo físico y natural, la investigación se propuso determinar a qué conjunto de alumnado (al que habla español como LM y/o al que habla un idioma diferente al español como LM) beneficia esta modalidad de ATAL.

Para este fin se determinó realizar un análisis -por separado- del rendimiento del alumnado con español como LM y del alumnado con una LM diferente al español, en función de su pertenencia a tutorías con modalidades diferenciadas de apoyo ATAL.

Como puede apreciarse en la Tabla 6, la información que se desprende del análisis descriptivo revela que, tanto el alumnado que habla español como LM como el aquél con una LM diferente al español en cuyas aulas se proporciona el apoyo ATAL de forma inclusiva consigue un rendimiento académico medio superior al obtenido por el alumnado en cuyas tutorías se suministra dicho apoyo fuera del aula de referencia; esta superioridad se constata en el conjunto de las competencias analizadas, con la única excepción de la expresión escrita, en relación a cuyo rendimiento el valor del promedio es el mismo para el alumnado cuya LM es el español (véase 4.1.1.).

Asimismo, los valores tanto de la mediana como de la moda son, en la mayoría de los casos, sensiblemente más elevados en el caso de ambos grupos de alumnos en los que el apoyo ATAL se realiza dentro de sus aulas de referencia.

En relación del alumnado que habla español como LM, estas diferencias de rendimiento se producen en el conjunto de las competencias analizadas, incluida la relativa a Comunicación Lingüística (con una diferencia de casi 3 puntos en el promedio obtenido por el alumnado que asiste a grupos de clases en los que se suministra apoyo ATAL de manera inclusiva frente al que pertenece a clases en que se realiza dicho apoyo fuera del aula), aunque resultan especialmente llamativas en Matemáticas (con una diferencia de más de 10 puntos en el promedio obtenido por el alumnado que asiste a grupos de clases en los que se suministra apoyo ATAL de manera inclusiva frente al que pertenece a clases en que se realiza dicho apoyo fuera del aula), y en algo más de 6 puntos en el caso del rendimiento en Conocimiento e interacción con el medio físico y natural. 
Ma Elvira Barrios Espinosa y Luis Morales Orozco Apoyo lingüístico inclusivo a alumnado...

La comparación de estos dos grupos de alumnado con español como LM con respecto a las medidas de rendimiento seleccionadas en la investigación mediante la prueba U de Mann-Whitney revela que no existen diferencias estadísticamente significativas entre ellos (Comunicación Lingüística: $p=0,926$; Comprensión oral: $p=0,555$; Comprensión lectora: $\mathrm{p}=0,587$; Expresión escrita: $\mathrm{p}=0,891$; Matemáticas: $\mathrm{p}=0,078$; Conocimiento e interacción con el medio físico y natural: $\mathrm{p}=0,285)$.

Las diferencias en redimiento entre grupos con apoyo ATAL dentro y fuera del aula son más destacadas incluso cuando se analizan los resultados obtenidos por alumnado cuya LM no es el español, y representan más de 7 puntos en Comunicación lingüística, más de 10 en Matemáticas y más de 9 , en Conocimiento e interacción con el medio físico y natural. Asimismo, en las tres dimensiones de comunicación lingüística evaluadas, en comprensión oral, comprensión lectora y expresión escrita, el grupo con apoyo inclusivo obtiene un rendimiento notablemente superior.

La comparación de estos dos grupos de alumnado que no tienen el español como LM con respecto a las medidas de rendimiento seleccionadas en la investigación mediante la prueba U de Mann-Whitney revela que existen diferencias estadísticamente significativas entre ellos en el conjunto de competencias y en dos de las tres dimensiones de Comunicación lingüística (Comunicación Lingüística: $\mathrm{p}=0,006$; Comprensión oral: $\mathrm{p}=0,027$; Comprensión lectora: $p=0,017$; Expresión escrita: $p=0,213$; Matemáticas: $p=0,005$; Conocimiento e interacción con el medio físico y natural: $\mathrm{p}=0,001$ ).

Se confirma, pues, conforme a los datos de este estudio, que el apoyo ATAL en un contexto inclusivo beneficia tanto a alumnado cuya lengua materna es el español como, de manera muy destacada, al alumnado para el que está concebido dicho apoyo ATAL, y que este beneficio parece ser mayor que el obtenido cuando este apoyo se realiza fuera del aula de referencia. El apoyo ATAL dentro del aula de referencia parece representar, por consiguiente, una modalidad más rentable y eficiente de destinar recursos humanos a este tipo de apoyo educativo del que se aprovecha, según los datos de nuestro estudio, tanto alumnado con dificultades lingüísticas como alumnado que no presenta estas dificultades. 
Tabla 6. Resultados del análisis estadístico descriptivo de las variables:

Rendimiento en la 'Prueba de competencias básicas en Comunicación lingüística, Rendimiento en la 'Prueba de competencia básica en Matemáticas' y Rendimiento en la 'Prueba de competencia básica en el Conocimiento y la interacción con el mundo físico y natural', en función de la LM del alumnado y la modalidad de apoyo ATAL de su grupo.

\begin{tabular}{|c|c|c|c|c|c|c|c|}
\hline & LM & $\begin{array}{c}\text { Tutoría } \\
\text { con apoyo } \\
\text { ATAL... }\end{array}$ & $\mathrm{N}$ & Media & Mediana & Moda & $\begin{array}{l}\text { Desv. } \\
\text { típica }\end{array}$ \\
\hline \multirow{4}{*}{ 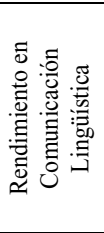 } & \multirow{2}{*}{$\sum_{\text {至 }}$} & $\begin{array}{l}\text { dentro del } \\
\text { aula }\end{array}$ & 10 & 64,50 & 65,50 & 67 & 7,561 \\
\hline & & $\begin{array}{l}\text { fuera del } \\
\text { aula }\end{array}$ & 10 & 61,70 & 64,00 & $52^{\mathrm{a}}$ & 14,190 \\
\hline & \multirow{2}{*}{ 吕蛭 } & $\begin{array}{l}\text { dentro del } \\
\text { aula }\end{array}$ & 23 & 51,22 & 53,00 & $33^{\mathrm{a}}$ & 9,945 \\
\hline & & $\begin{array}{l}\text { fuera del } \\
\text { aula }\end{array}$ & 24 & 43,58 & 41,00 & $37^{\mathrm{a}}$ & 9,468 \\
\hline \multirow{4}{*}{ 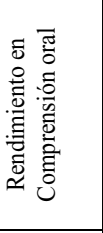 } & \multirow{2}{*}{ 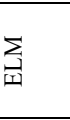 } & $\begin{array}{l}\text { dentro del } \\
\text { aula }\end{array}$ & 10 & 3,70 & 4,00 & 4 & ,949 \\
\hline & & $\begin{array}{l}\text { fuera del } \\
\text { aula }\end{array}$ & 10 & 3,40 & 3,00 & 3 & 1,075 \\
\hline & \multirow{2}{*}{ 之忩 } & $\begin{array}{l}\text { dentro del } \\
\text { aula }\end{array}$ & 23 & 2,43 & 3,00 & 3 & 1,080 \\
\hline & & $\begin{array}{l}\text { fuera del } \\
\text { aula }\end{array}$ & 24 & 1,75 & 1,50 & 1 & ,897 \\
\hline \multirow{4}{*}{ 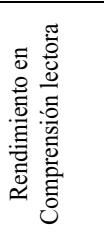 } & \multirow{2}{*}{ 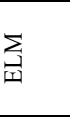 } & $\begin{array}{l}\text { dentro del } \\
\text { aula }\end{array}$ & 10 & 3,40 & 3,00 & 3 & ,699 \\
\hline & & $\begin{array}{l}\text { fuera del } \\
\text { aula }\end{array}$ & 10 & 3,00 & 3,00 & 4 & 1,054 \\
\hline & \multirow{2}{*}{ 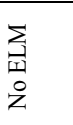 } & $\begin{array}{l}\text { dentro del } \\
\text { aula }\end{array}$ & 23 & 2,65 & 3,00 & 3 & ,885 \\
\hline & & $\begin{array}{l}\text { fuera del } \\
\text { aula }\end{array}$ & 24 & 2,17 & 2,00 & 2 & ,761 \\
\hline \multirow{4}{*}{ 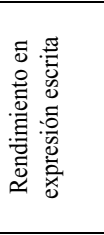 } & \multirow{2}{*}{$\sum_{\text {至 }}$} & $\begin{array}{l}\text { dentro del } \\
\text { aula }\end{array}$ & 10 & 2,90 & 3,00 & 4 & 1,101 \\
\hline & & $\begin{array}{l}\text { fuera del } \\
\text { aula }\end{array}$ & 10 & 2,90 & 4,00 & 4 & 1,449 \\
\hline & \multirow{2}{*}{ 吕壆 } & $\begin{array}{l}\text { dentro del } \\
\text { aula }\end{array}$ & 23 & 1,65 & 1,00 & 1 & ,775 \\
\hline & & $\begin{array}{l}\text { fuera del } \\
\text { aula }\end{array}$ & 24 & 1,42 & 1,00 & 1 & ,776 \\
\hline \multirow{4}{*}{ 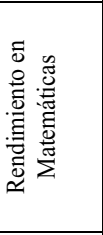 } & \multirow{2}{*}{$\sum_{\text {至 }}$} & $\begin{array}{l}\text { dentro del } \\
\text { aula }\end{array}$ & 10 & 50,80 & 55,50 & 43 & 12,752 \\
\hline & & $\begin{array}{l}\text { fuera del } \\
\text { aula }\end{array}$ & 10 & 40,60 & 37,50 & 26 & 13,352 \\
\hline & \multirow{2}{*}{ 之 $\sum_{i=1}$} & $\begin{array}{l}\text { dentro del } \\
\text { aula }\end{array}$ & 18 & 44,11 & 44,50 & 36 & 11,995 \\
\hline & & $\begin{array}{l}\text { fuera del } \\
\text { aula }\end{array}$ & 23 & 34,09 & 31,00 & $23^{\mathrm{a}}$ & 10,740 \\
\hline \multirow{4}{*}{ 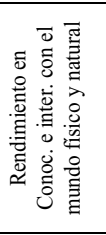 } & \multirow{2}{*}{ 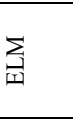 } & $\begin{array}{l}\text { dentro del } \\
\text { aula }\end{array}$ & 9 & 56,78 & 56,00 & 54 & 8,273 \\
\hline & & $\begin{array}{l}\text { fuera del } \\
\text { aula }\end{array}$ & 10 & 50,00 & 54,50 & $34^{\mathrm{a}}$ & 11,757 \\
\hline & \multirow{2}{*}{ 设公 } & $\begin{array}{l}\text { dentro del } \\
\text { aula }\end{array}$ & 23 & 47,65 & 48,00 & $40^{\mathrm{a}}$ & 9,717 \\
\hline & & $\begin{array}{l}\text { fuera del } \\
\text { aula }\end{array}$ & 23 & 38,09 & 35,00 & 34 & 9,380 \\
\hline
\end{tabular}


Ma Elvira Barrios Espinosa y Luis Morales Orozco Apoyo lingüístico inclusivo a alumnado...

Entendemos que la divulgación de estos resultados, en los que se evidencia la mayor eficiencia de la modalidad inclusiva de apoyo ATAL (frente a la modalidad segregacionista) para procurar la adquisición de competencias curriculares, la cual beneficia tanto al alumnado autóctono hispanohablante como al alumnado no hispanohablante con la misma asignación de recursos humanos, junto a la difusión del enfoque didáctico y del modelo adoptado de organización y de colaboración entre profesorado, pueden modificar percepciones de padres y de profesorado sobre la repercusión académica negativa de la presencia de alumnado no hispanohablante en las aulas, puestas de manifiesto en el estudio de Ortiz (2008). Asimismo, estamos convencidos de que el trabajo colaborativo en una misma aula de profesorado de apoyo y profesorado ordinario fomenta, tanto el que este último asuma la responsabilidad del alumnado extranjero en sus clases y que no la delegue en el docente adscrito al programa de ATAL, situación relativamente frecuente en centros escolares y que ha sido evidenciada en algunos estudios (García Fernández y Moreno, 2007; García Fernández et al., 2007; Ortiz, 2008), como el que se satisfaga la demanda del profesorado ordinario de que el apoyo ATAL se amplíe para facilitar la adquisición de contenidos curriculares diferentes a los lingüísticos (Ortiz, 2008; Porras, García García y Cotrina, 2009). Coincidimos, en este sentido, con Porras, García y Cotrina (2009: 13) cuando afirman:

[...] el ATAL, como programa, y el maestro o maestra de ATAL, como profesional educativo, poseen un valor incalculable como herramientas para facilitar aulas inclusivas a través de un modelo colaborativo de acción didáctica en el aula.

\section{Conclusiones}

Aunque somos conscientes de la limitación que representa que este estudio se circunscriba a un único centro y que los resultados obtenidos no sean generalizables a otros contextos, sí que poseen un enorme valor por cuanto que, por primera vez, a) se cifra en términos de rendimiento académico o adquisición de competencias curriculares una modalidad inclusiva de apoyo ATAL frente a la modalidad imperante de apoyo externo y b) los resultados apuntan a que una modalidad de apoyo ATAL inclusivo sostenido durante varios cursos académicos puede repercutir más positivamente en el rendimiento académico del grupo de clase en su conjunto en el que se desarrolla que la modalidad de apoyo fuera del aula.

Asimismo, según los datos de rendimiento académico de los que ha hecho uso este estudio, aunque es el alumnado para el que se concibe el apoyo lingüístico ATAL el principal beneficiario de una modalidad inclusiva de esta ayuda, el alumnado cuya lengua materna es el español se beneficia asimismo de forma notable de este apoyo dentro del aula.

Se constata, por tanto, que el alumnado con lengua materna diferente al español es capaz de adquirir la competencia suficiente en esta lengua como lengua vehicular del aprendizaje del currículo sin necesidad de salir de su aula de referencia por motivo alguno y de integrarse de forma efectiva a la rutina escolar desde su incorporación al centro. 
En consecuencia, los hallazgos de este estudio demuestran la mayor rentabilidad y eficiencia de una modalidad inclusiva de apoyo lingüístico a alumnado inmigrante de lengua materna diferente al español a efectos de favorecer el aprendizaje de contenidos curriculares -no solo en alumnado inmigrante con dificultades en la lengua vehicular sino, también, en alumnado autóctono. Representa, a este respecto, un argumento de peso para que la Administración Educativa no solo recomiende, sino que procure la aplicación efectiva y generalizada -salvo casos auténticamente excepcionales- de esta modalidad inclusiva de apoyo lingüístico frente a la que supone apartar al alumnado del seguimiento del currículo ordinario en su aula de referencia durante unas horas a la semana.

Por último, entendemos que es imprescindible que los profesionales de la educación afronten el fenómeno imparable de la diversidad de origen lingüístico del alumnado en nuestros centros escolares como un desafío enriquecedor porque, como afirma Cummins (2001: 20):

[...] el capital cultural, lingüístico e intelectual de nuestras sociedades se incrementará significativamente cuando dejemos de considerar a los niños y niñas cultural y lingüísticamente diversos como 'un problema que resolver' y, por el contrario, abramos nuestros ojos a los recursos lingüísticos, culturales e intelectuales que estos niños y niñas traen consigo de sus casas a nuestras escuelas y a nuestras sociedades. $^{9}$

\section{REFERENCIAS}

Baker, C. (2001): Foundations of Bilingual Education and Bilingualism. Third Edition. Philadelphia. PA: Multilingual Matters Ltd.

Barrios Espinosa, M.E. y García Mata, J. (2006): Formación de Didáctica de Lenguas Extranjeras a través de la observación en el aula. Málaga: Universidad de Málaga.

Bonal, X. (2004). «Escola. La inmigració a debat: diversitat i ordenament jurídic», en Debats, 3: 7-17.

Cummins, J. (2000): Language, Power and Pedagogy. Bilingual Children in the Crossfire. Clevedon: Multilingual Matters.

Cummins, J. (2001): «Bilingual Children's Mother Tongue: Why is it important for education?», en SPROGFORUM, 19: 15-20.

Díaz-Aguado, M.J. (2006): Educación intercultural y aprendizaje cooperativo. Madrid: Pirámide.

García Fernández, J.A., Moreno Herrero, I. (2003). La respuesta a las necesidades educativas de los hijos de inmigrantes en la Comunidad de Madrid. Madrid: Consejo Económico y Social de Madrid.

García Fernández, J.A., Moreno Herrero, I. y Sánchez Delgado P. (2008). «Las aulas de enlace madrileñas, ¿espacios para el aprendizaje de la lengua vehicular o burbujas de acogida?». Girona: Comunicación en el Sexto Simposio de Lengua, Cultura e Inmigración. Disponible en http://web.udg.edu/ice/simposi/doc/re_jgarcia.pdf. Consultado el 12 de octubre de 2009.

\footnotetext{
9 Traducción de los autores.
} 
Ma Elvira Barrios Espinosa y Luis Morales Orozco Apoyo lingüístico inclusivo a alumnado...

García Fernández, J.A., Moreno Herrero, I., Sánchez Delgado P., García Medina, R., Goenechea Permisán, C., Cluse Chapa, C., Placarte, I., Salas Villegas, M.A. (2007). Informe parcial de la investigación "Estudio del sistema y funcionamiento de las aulas de enlace madrileñas». Disponible en www.ucm.es/info/indice/Informe\%20Fase\%201.pdf. Consultado el 7 de marzo de 2011.

García Marín, C. y Martínez Ten, A. (2004). El juego de las cuatro esquinitas del mundo. Libro de juegos para favorecer las relaciones interculturales. Madrid: Los libros de la Catarata.

Jiménez Gámez, R. (2002). «Multiculturalidad y educación: escenarios de investigación y acción en la provincia de Cádiz», en L. Serra Salomón (ed.), Inmigración extranjera en Andalucía. II Seminario sobre la investigación de la inmigración extranjera en Andalucía (195-210). Sevilla: Consejería de Gobernación. Dirección General de Coordinación de Políticas Migratorias.

Lambert, W.E. (1975): Culture and Language as Factors in Learning and Education. Toronto: OISE Press.

Martín Rojo, L. (1995): «Bilingüismo y diversidad cultural en el aula», en Didáctica, 7: 309-320.

Martín Rojo, L. (Dir.), Alcalá, E., Gari, A., Gari, L., Sierra, I. y Rodríguez, M.A. (2004): ¿Asimilar o integrar? Dilema ante el multilingüismo en las aulas. Madrid: Ministerio de Educación y Ciencia, Secretaría General de Educación.

Morales Orozco, L. (2006): La integración lingüistica del alumnado inmigrante. Propuestas para el aprendizaje cooperativo. Madrid: Los libros de la Catarata.

Morales Orozco, L. (2007): «Cómo integrar lingüísticamente a alumnado inmigrante», en Intercultualidad en la escuela, 60: 34-36.

Morales Orozco, L. (2009): «Bilingüismo aditivo o de ganancia en aulas inclusivas», en Barrios Espinosa, M.E. (ed.): La educación bilingüe en Andalucia: Reflexiones, experiencias y propuestas (pp. 165-178). Granada: Grupo Editorial Universitario.

Morales Orozco, L. Barrios Espinosa, M.E. y Felipe Morales, A. (2009). Memoria final del proyecto de investigación educativa «Integración lingüistica y bilingüismo de ganancia en contextos multilingües e inclusivos». Documento no publicado.

Ortiz Cobo, M. (2006). «Mecanismos de transmisión del español como segunda lengua en contextos escolares de inmigración», en Educación y Futuro, 15: 91-108.

Ortiz Cobo, M. (2007). «Segunda Lengua como medio de integración escolar», en Revista Iberoamericana de Educación, 41, 4: 1-5.

Ortiz Cobo, M. (2008). «Inmigración en las aulas: percepciones prejuiciosas de los docentes», en Papers, 87: 253-268.

Porras Vallejo, R., García García, M. y Cotrina García, M.J. (2009). «Posibilidades y límites del programa de ATAL en el marco de una escuela inclusiva», en Revista de Educación Inclusiva, 2, 1: 11-28.

Quintana, A. (2003). «Estrategias de acogida y acompañamiento en ESO: una propuesta de itinerario con alumnado de incorporación tardía», en Aula de Innovación Educativa, 126: 53-57

Stainback, S. y Stainback, W. (1999): Aulas inclusivas. Madrid: Narcea.

Trujillo Sáez, F. (2006): Cultura, comunicación y lenguaje. Reflexiones para la enseñanza de la lengua en contextos multiculturales. Granada: Octaedro Andalucía. 